\title{
De FUTURE trial; een multicenter RCT naar MRI-geleide prostaatbiopten
}

\section{Vergelijking van drie technieken van target biopten en vergelijking met systematische biopten}

\author{
O. Wegelin ${ }^{1}$ L. Exterkate ${ }^{2}$ J. O. Barentsz ${ }^{3}$ M. G. van der Leest ${ }^{3} \cdot$ J. C. Kelder ${ }^{1} \cdot$ J. A. Kummer ${ }^{1}$. W. Vreuls ${ }^{2}$. \\ P. C. de Bruin' - T. F. de Vocht ${ }^{4}$ J. E. Nuininga ${ }^{5}$ J. L. H. R. Bosch ${ }^{6}$ D. M. Somford ${ }^{2}$ H. H. E. van Melick ${ }^{1}$
}

Published online: 15 May 2019

(c) The Author(s) 2019

\section{Samenvatting}

De FUTURE-trial is een multicenter gerandomiseerde studie naar drie technieken van MRI-geleide target biopten (TB's) bij patiënten na negatieve systematische biopten (SB's) en blijvende verdenking op prostaatkanker. Tussen 2014 en 2017 ondergingen 665 patiënten een multiparametrische MRI. 234 (35\%) patiënten met een PIRADS-laesie $\geq 3$ werden 1:1:1 gerandomiseerd voor TB: in-bore MRI (MRI-TB), MRI/TRUS-fusie (FUS-TB), en cognitieve fusie (COG-TB). Na FUS-TB en COG-TB werden tevens SB's afgenomen. Tussen de drie technieken waren geen significante verschillen in detectie van prostaatkanker en significante prostaatkanker (MRI-TB 54,5\%/32,5\%, FUS-TB 49,4\%/34,2\%, en COG-TB $43,6 \% / 33,3 \%, p=0,39 / p=0,98$ ). De combinatie van SB's en TB's versus enkel TB's zorgde voor $1 \%$ meer detectie van significante prostaatkanker (35\% versus $34 \%$ ). Er was geen significant voordeel voor één TB-techniek voor het detecteren van (klinisch significante) prostaatkanker. De toegevoegde waarde van een SB was beperkt.

Trefwoorden MRI $\cdot$ prostaatkanker $\cdot$ diagnostiek $\cdot$ biopten

\section{The FUTURE trial: a multicenter RCT on MRI-guided prostate biopsies}

Comparison of three techniques of targeted biopsies and comparison with systematic biopsies

\begin{abstract}
The FUTURE trial is a multicenter RCT on 3 MRI-based target biopsy (TB) techniques among patients with negative systematic biopsies (SBs) and persisting suspicion of prostate cancer. Between 2014 and 2017665 patients underwent multiparametric MRI. 234 (35\%) patients with a PIRADS $\geq 3$ lesion were randomised 1:1:1 for TB: in-bore MRI (MRI-TB), MRI/ultrasound fusion (FUS-TB), and cognitive fusion (COG-TB). After FUS-TB and COG-TB additional SB was performed. No significant differences in prostate cancer detection and significant prostate cancer were found (MRI-TB $54.5 \% / 32.5 \%$, FUS-TB 49.4\%/34.2\%, and COG-TB 43.6\%/33.3\%, $p=0.39 / p=0.98$ ). Combined SBs and TBs versus TBs alone resulted in $1 \%$ more csPCa detection ( $35 \%$ vs $34 \%$ ). There was no significant advantage of one TB technique for the detection of overall prostate cancer, nor for clinically significant prostate cancer. The additional value of SBs was limited.
\end{abstract}

Keywords MRI $\cdot$ prostate cancer $\cdot$ diagnosis $\cdot$ biopsy

Gedeelde eerste auteur: O. Wegelin, L. Exterkate

Gedeelde laatste auteur: D.M. Somford, H.H.E. van Melick

Het huidige manuscript is geschreven op uitnodiging.

drs. L. Exterkate

1.exterkate@cwz.nl

1 St. Antonius Ziekenhuis, Utrecht/Nieuwegein, Nederland
2 Afdeling urologie, Canisius-Wilhelmina Ziekenhuis, Nijmegen, Nederland

3 Radboudumc, Nijmegen, Nederland

4 Bernhoven, Uden, Nederland

5 Ziekenhuis Rivierenland, Tiel, Nederland

6 UMC, Utrecht, Nederland 


\section{Introductie}

Prostaatkanker is de meest voorkomende kanker bij mannen in Nederland [1]. De standaard diagnostiek naar prostaatkanker bestaat uit het onderzoeken van transrectaal echogeleide (TRUS) systematische biopten (SB's) [2]. Conventionele TRUS is niet in staat prostaatkanker betrouwbaar te onderscheiden van benigne prostaatweefsel [3]. De huidige richtlijn adviseert een multiparametrische (mp)MRI te verrichten bij patiënten met blijvende klinische verdenking op prostaatcarcinoom. Indien op mpMRI suspecte afwijkingen worden gezien, kunnen er target biopten (TB's) van deze laesies verricht worden, al dan niet in combinatie met herhaalde SB's [2].

Momenteel zijn er voor het afnemen van TB's drie verschillende technieken beschikbaar. Allereerst kunnen biopten via MRI (MRI-TB) worden afgenomen onder directe MRI-geleiding. Een alternatief is MRI-TRUS-fusie (FUSTB), waarbij MRI-beelden middels software worden gefuseerd met TRUS-beelden en op deze wijze biopten uit de afwijking kunnen worden genomen. Ten slotte kunnen cognitieve biopten (COG-TB) genomen worden, waarbij de uroloog op de hoogte is van de locatie van de afwijking op MRI, en gericht biopten neemt van het gebied. Er bestaat geen consensus over welke techniek de voorkeur verdient.

De FUTURE trial is een multicenter gerandomiseerde studie die de drie TB-technieken met elkaar vergelijkt. Data van de FUTURE trial werden (ten dele) eerder gepubliceerd of worden momenteel voorbereid voor publicatie [4]. Dit artikel betreft een subanalyse van de uitkomsten van TB's met herhaalde SB's.

\section{Materiaal}

\section{Studieopzet en populatie}

De FUTURE trial heeft tussen 2014 en 2017 in twee nietacademische opleidingsziekenhuizen in Nederland patiënten gerekruteerd, met verwijzingen uit omliggende ziekenhuizen. Het protocol werd geregistreerd bij het Nederlands Trial Register (NTR4988). Voorafgaand aan inclusie heeft medisch-ethische goedkeuring plaatsgevonden. Alle deelnemers tekenden bij inclusie informed consent.

Inclusiecriteria waren ten minste eenmaal eerder negatieve SB's in de afgelopen vier jaar en blijvende klinische verdenking op prostaatkanker (op basis van PSA $\geq 4 \mathrm{ng} / \mathrm{ml}$ en/of een afwijkend rectaal toucher). Exclusiecriteria waren eerder gediagnosticeerd prostaatkanker, eerdere TB-procedures, een bewezen urineweginfectie, contra-indicaties voor mpMRI of TB, mpMRI of biopten die niet uitgevoerd werden volgens protocol, of intrekken van consent.

\section{MRI}

Deelnemers ondergingen een 3 Tesla mpMRI volgens PIRADS-v2-criteria [5, 6]. Beeldvorming bestond uit T2-gewogen opnames (T2W), diffusiegewogen opnamen (DWI) en contrastversterkte opnamen (DCE). Er werd intraveneus buscopan- en gadoliniumcontrast gebruikt. De mpMRIbeelden werden centraal beoordeeld door een van twee expert uroradiologen met 20 en 5 jaar ervaring in het lezen van prostaat-MRI's middels PIRADS-v2 (appendix 1). De radiologen werden niet geblindeerd voor klinische gegevens conform de klinische praktijk.

\section{Randomisatie}

Deelnemers met een PIRADS-laesie $\geq 3$ op mpMRI werden 1:1:1 gerandomiseerd voor het ondergaan van TB's middels MRI-TB, FUS-TB of COG-TB. Indien er op mpMRI geen suspecte afwijking (PIRADS $\leq 2$ ) werd gezien, ondergingen patiënten biochemische follow-up middels PSAmetingen.

\section{Biopten en histopathologie}

Rondom de biopten kregen alle patiënten antibiotische profylaxe bestaande uit drie dagen ciprofloxacine $(500 \mathrm{mg}$ tweemaal daags). Patiënten in de FUS-TB-groep ontvingen een klysma voorafgaand aan de procedure. Anticoagulantia werden gecontinueerd met uitzondering van coumarinederivaten.

MRI-TB's werden verricht in de MRI-scanner (Magnetom Skyra ${ }^{\circledR}$ Siemens). Suspecte afwijkingen werden transrectaal aangeprikt met een MRI-compatibele biopsienaald. Adequate naaldpositie werd gecontroleerd middels MRI-beelden. MRI-TB's werden verricht door een team van 10 getrainde promovendi.

FUS-TB's werden verricht op de operatiekamer middels een transperineaal MRI/TRUS-fusiesysteem (Biopsee ${ }^{\circledR}$ Medcom) onder algehele of spinale anesthesie in steensnedeligging. Axiale T2W-beelden werden geïmporteerd in het systeem. 3D TRUS werd verricht en softwarematig werden axiale T2W-MRI-beelden en 3D TRUS-beelden rigide gefuseerd. Biopten werden transperineaal genomen onder MRI/TRUS-fusiegeleiding door een team van vijf getrainde urologen of promovendi.

COG-TB's werden poliklinisch genomen middels TRUS (Hitachi Hi-Vision Preirus ${ }^{\circledR}$ of BK Pro-Focus ${ }^{\circledR}$ ) nadat de MRI-beelden voorafgaand aan de biopten opnieuw werden bekeken door de biopteur. COG-TB's werden verricht door een team van vijf getrainde urologen of promovendi. In de FUS-TB- en COG-TB-groepen werden TB's gevolgd door herhaalde SB's middels een vast schema (inclusief ten minste twee anterieure en twee transitiezonebiopten) onafhan- 
Figuur 1 Stroomdiagram van de studie

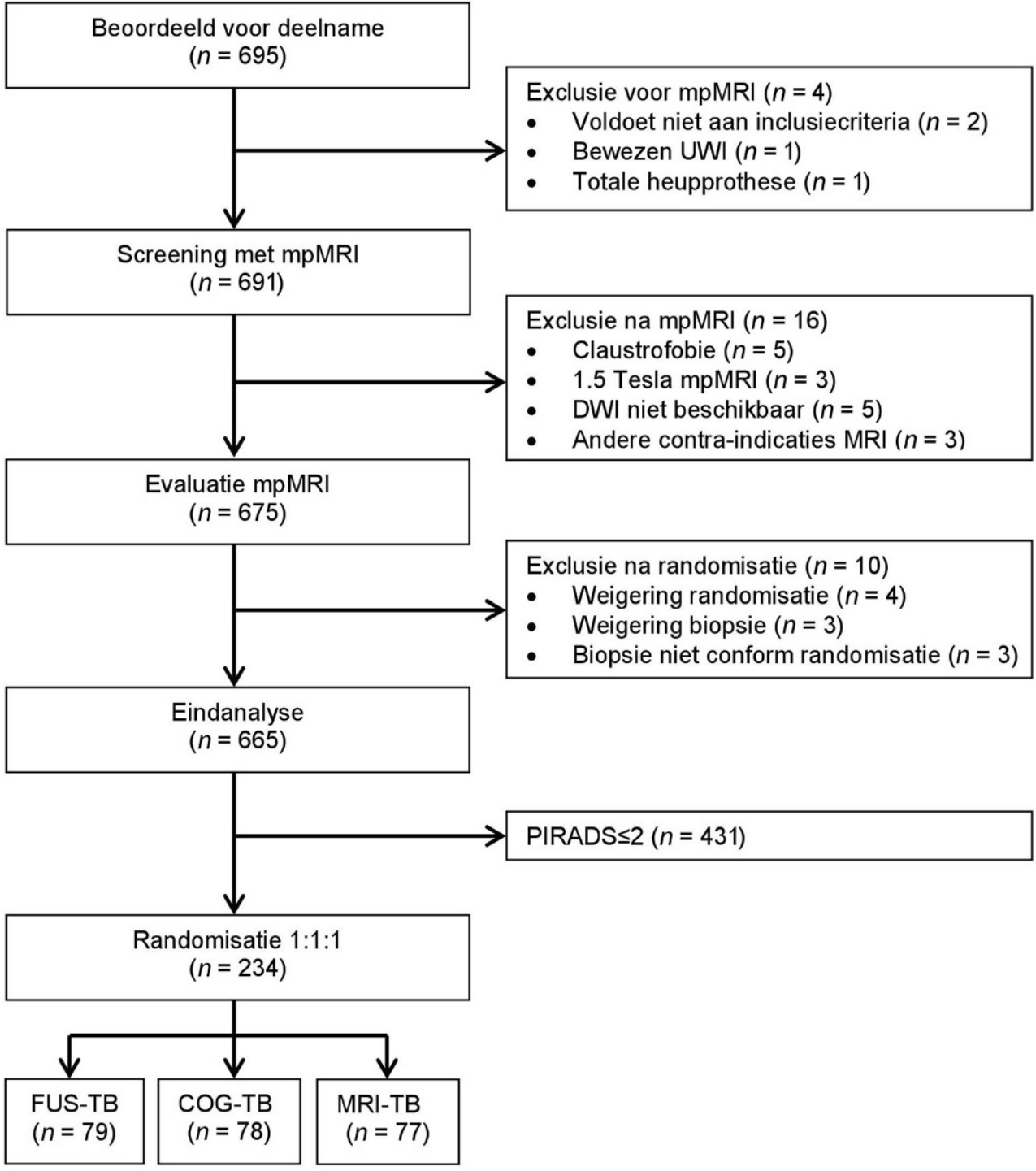

kelijk van de locatie van de suspecte laesies op mpMRI. Het aantal SB's was afhankelijk van het prostaatvolume (volume $\leq 40 \mathrm{ml}=8$ biopten; volume $40-60 \mathrm{ml}=10$ biopten; volume $\geq 60 \mathrm{ml}=12$ biopten) (appendix 2). In de MRI-TBgroep was het technisch niet mogelijk om herhaalde SB's uit te voeren. Deze patiënten werden buiten beschouwing gelaten in de vergelijking tussen TB en SB.

De biopten werden voor iedere laesie separaat verwerkt volgens ISUP-criteria en beoordeeld door een uropatholoog per deelnemend centrum (met 10, 11 en 17 jaar ervaring in prostaatkankerdiagnostiek) [7]. De biopsie-uitkomsten van SB- en TB-procedures werden separaat gerapporteerd. De patholoog was geblindeerd voor de gebruikte TB-techniek. Klinisch significante prostaatkanker werd gedefinieerd als elke Gleason-score $\geq 3+4=7$.

\section{Statistiek}

De primaire uitkomstmaat was de detectie van prostaatkanker per TB-techniek. Secundaire uitkomstmaten waren de detectie van klinisch-significante prostaatkanker, uitkomsten van herhaalde SB's, patiënt- en mpMRI-karakteristieken, en procedurele uitkomsten.

We veronderstelden dat FUS-TB's een vergelijkbare prostaatkankerdetectie zouden hebben als MRI-TB's (noninferiority analyse) en dat zowel MRI-TB's als FUS-TB's een betere detectie zouden hebben dan COG-TB's (superiority analyse). De groepsgrootte werd berekend op basis van geschatte tumordetectie van $40 \%$ voor zowel MRI-TB als FUS-TB en van $25 \%$ voor COG-TB, en een opbrengst van $69 \%$ van PIRADS-laesie $\geq 3$ op mpMRI na eerdere negatieve biopten [8-10].

Voor de non-inferiority analyse werd een groepsgrootte van 131 per groep berekend en voor de superiority analyse 
Hier staat een advertentie.

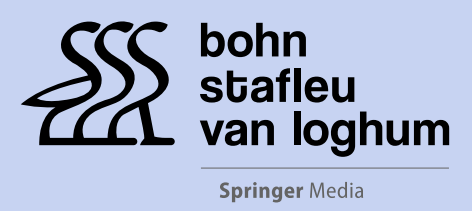

Houten 2019 
Hier staat een advertentie.

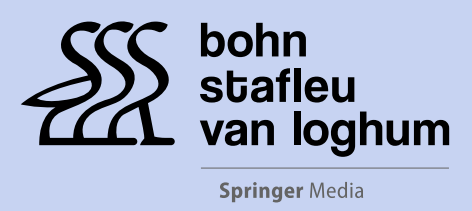

Houten 2019 
een groepsgrootte van 152 per groep. Om de randomisatie te faciliteren, werd een equivalente groepsgrootte van 152 per groep gebruikt, resulterend in 456 patiënten voor alle groepen gecombineerd. Om te corrigeren voor verwachte uitval werden 10 additionele patiënten berekend. Uitgaande van een opbrengst van $69 \%$ van PIRADS-laesies $\geq 3$ op mpMRI bij deze patiëntencategorie waren in totaal 675 deelnemers noodzakelijk.

Patiënt- en mpMRI-karakteristieken werden met elkaar vergeleken middels one-way ANOVA, Kruskal-Wallis en chi-kwadraattoetsen. De detectie-uitkomsten van prostaatkanker en significant prostaatkanker tussen de drie TBgroepen werden vergeleken middels de chi-kwadraattoets.

De uitkomsten van herhaalde SB's en TB's (FUS-TB en COG-TB) werden vergeleken met McNemar's test voor zowel prostaatkanker als significant prostaatkanker. De sensitiviteit van TB's en SB's werd berekend als het aantal positieve uitkomsten van TB's of SB's gedeeld door het totale aantal positieve uitkomsten (combinatie TB's en SB's). De specificiteit van TB's en SB's werd berekend als het aantal negatieve uitkomsten van TB's of SB's gedeeld door het totale aantal negatieve uitkomsten (combinatie TB's en SB's). Alle statistische analyses werden uitgevoerd met SPSS en overal werd een $5 \%$ significantie toegepast.

\section{Resultaten}

\section{Populatie}

Er werden 695 patiënten gerekruteerd. Gedurende de studie werden 30 patiënten om verschillende redenen geëxcludeerd (fig. 1) resulterend in een totaal van 665 geïnclu- deerde patiënten. Bij 234 van de 665 geïncludeerde patiënten werden op mpMRI in totaal 263 PIRADS-laesies $\geq 3$ gevonden. Deze 234 patiënten ondergingen 1:1:1 randomisatie; 77 voor MRI-TB, 79 voor FUS-TB, en 78 voor COG-TB. De patiënt- en mpMRI-karakteristieken van de 234 patiënten staan weergegeven in tab. 1. De overige 431 patiënten met PIRADS-laesies $\leq 2$ werden biochemisch vervolgd.

\section{Vergelijking van de drie TB-groepen}

Er werden geen significante verschillen gevonden in patiënt- en mpMRI-karakteristieken tussen de drie groepen (tab. 2). Ook werden tussen de groepen geen significante verschillen gevonden in prostaatkankerdetectie (MRI-TB $54,5 \%$, FUS-TB 49,4\% en COG-TB 43,6\%; $p=0,39$; tab. 3). Het verschil in prostaatkankerdetectie tussen FUS-TB en MRI-TB was -5,2\% (95\%-BI=-20,6-10,5; $p=0,52)$. Het verschil in prostaatkankerdetectie tussen FUS-TB en COG-TB was 5,8\% (95\%-BI=-9,8-21,1; $p=0,47)$. Het verschil in prostaatkankerdetectie tussen COG-TB en MRI-TB was $-11,0 \%$ (95\%-BI $=-26,2-4,8$; $p=0,17)$. De non-inferiorityanalyse tussen MRI-TB en FUS-TB was niet conclusief (aangezien de onderste limiet van het $95 \%$-BI kleiner is dan -15\%). Zowel MRI-TB als FUS-TB waren niet significant superieur ten opzichte van COG-TB ( $p$-waarden van 0,17 en 0,47 , respectievelijk).

Er werden geen significante verschillen gevonden in de detectie van significante prostaatkanker tussen de drie groepen (MRI-TB 32,5\%, FUS-TB 34,2\%, en COG-TB $33,3 \%, p=0,98$ ) (tab. 3). Het verschil in significante prostaatkankerdetectie tussen FUS-TB en MRI-TB was $1,7 \%$ $(95 \%-\mathrm{BI}=-13,1-16,4 ; p=0,82)$. Het verschil in signifi-
Tabel 1 Baseline patiënt- en mpMRI-karakteristieken van gebiopteerde cohort

\begin{tabular}{lc}
\hline & $\begin{array}{c}\text { cohort met PIRADS } \geq 3 \text { op mpMRI } \\
(n=234)\end{array}$ \\
\hline leeftijd, gem. (SD) & $65,7(6,4)$ \\
PSA in ng/ml, gem. (SD) & $11,2(8,5)$ \\
volume bij TRUS in ml, gem. (SD) & $47,4(17,7)$ \\
klinisch stadium (RT), No. (\%) & \\
cT1c & $188(80,3)$ \\
cT2a/b & $40(17,1)$ \\
cT2c & $3(1,3)$ \\
cT3a & $3(1,3)$ \\
aantal eerdere negatieve biopten & $1(1-2)$ \\
mediaan (IQR) & \\
maanden tussen laatste biopt en mpMRI & $8(4-23)$ \\
mediaan (IQR) & \\
mpMRI-uitkomst, No. (\%) & $64(27,4)$ \\
PIRADS 3 & $101(43,2)$ \\
PIRADS 4 & $69(29,5)$ \\
PIRADS 5 &
\end{tabular}


Tabel 2 Baseline patiënt- en mpMRI-karakteristieken per TB-techniek

\begin{tabular}{|c|c|c|c|}
\hline & $\begin{array}{l}\text { FUS-TB } \\
(n=79)\end{array}$ & $\begin{array}{l}\text { COG-TB } \\
(n=78)\end{array}$ & $\begin{array}{l}\text { MRI-TB } \\
(n=77)\end{array}$ \\
\hline leeftijd, gem. (SD) & $64,6(\mathrm{SD} 6,9)$ & $66,5(\mathrm{SD} 6,3)$ & $66,0($ SD 5,9) \\
\hline PSA in $\mathrm{ng} / \mathrm{ml}$, gem. (SD) & $11,6(\mathrm{SD} 9,0)$ & $11,0(\mathrm{SD} 7,1)$ & $11,0(\mathrm{SD} 9,4)$ \\
\hline volume bij TRUS in ml, gem. (SD) & $45,4(\mathrm{SD} 14,4)$ & $48,5(\mathrm{SD} 18,1)$ & $48,3(\mathrm{SD} 20,2)$ \\
\hline \multicolumn{4}{|l|}{ klinisch stadium (RT), No. (\%) } \\
\hline cT0 & $62(78,5 \%)$ & $64(82,1 \%)$ & $62(80,5 \%)$ \\
\hline $\mathrm{cT} 2 \mathrm{a} / \mathrm{b}$ & $16(20,3 \%)$ & $12(15,4 \%)$ & $12(15,6 \%)$ \\
\hline $\mathrm{cT} 2 \mathrm{c}$ & $0(0 \%)$ & $2(2,6 \%)$ & $1(1,3 \%)$ \\
\hline cT3a & $1(1,3 \%)$ & $0(0 \%)$ & $2(2,6 \%)$ \\
\hline \multicolumn{4}{|l|}{ aantal eerdere negatieve biopten } \\
\hline mediaan (IQR) & $1(\mathrm{IQR} 1-1)$ & 1 (IQR 1-2) & 1 (IQR 1-2) \\
\hline \multicolumn{4}{|l|}{ maanden tussen laatste biopt en mpMRI } \\
\hline mediaan (IQR) & 8 (IQR 3-23) & 7 (IQR 4-23) & 9 (IQR 4-25) \\
\hline \multicolumn{4}{|l|}{ mpMRI-uitkomst, No. (\%) } \\
\hline PIRADS 3 & $23(29,1 \%)$ & $21(26,9 \%)$ & $20(26,0 \%)$ \\
\hline PIRADS 4 & $34(43,0 \%)$ & $32(41,0 \%)$ & $35(45,5 \%)$ \\
\hline PIRADS 5 & $22(27,8 \%)$ & $25(32,1 \%)$ & $22(28,6 \%)$ \\
\hline
\end{tabular}

\begin{tabular}{llccc}
\hline & $\begin{array}{l}\text { FUS-TB } \\
(n=79)\end{array}$ & $\begin{array}{l}\text { COG-TB } \\
(n=78)\end{array}$ & $\begin{array}{l}\text { MRI-TB } \\
(n=77)\end{array}$ & \\
\hline $\begin{array}{l}\text { detectiepercentage prostaatkanker } \\
\begin{array}{l}\text { No. }(\%) \\
\text { detectiepercentage significante prostaatkanker }\end{array}\end{array}$ & $39(49,4)$ & $34(43,6)$ & $42(54,5)$ & $p=0,39$ \\
$\begin{array}{l}\text { (Gleason-score } \geq 3+4=7), \text { No. }(\%) \\
\text { biopten }\end{array}$ & $27(34,2)$ & $26(33,3)$ & $25(32,5)$ & $p=0,98$ \\
$\begin{array}{l}\text { per patiënt, mediaan (IQR) } \\
\text { percentage positieve biopten (SD) }\end{array}$ & $4(3-5)$ & $3(3-4)$ & $2(2-3)$ & $p<0,05$ \\
\hline
\end{tabular}

Tabel 3 Vergelijking uitkomsten van drie TB-technieken

\section{Vergelijking TB's en SB's}

Totaal 152 patiënten ondergingen zowel TB's (76 FUS-TB en 76 COG-TB) als SB's.

De detectie van prostaatkanker middels TB's was $47 \%$ (71/152) en middels SB's 32\% (49/152) (tab. 5). Dit verschil van $15 \%$ was significant $(p<0,001 ; 95 \%$-BI $=6-22)$. Werden SB's en TB's gecombineerd, dan werd prostaatkanker in $53 \%$ (81/152) van de gevallen gedetecteerd, resulterend in $6 \%$ verschil in prostaatkankerdetectie ten opzichte van TB alleen. De sensitiviteit voor de detectie van prostaatkanker van TB's is 0,88 en van SB's is deze 0,60 ; de specificiteit voor prostaatkanker is 0,72 en 0,91 respectievelijk.

De detectie van significante prostaatkanker middels TB's was $34 \%$ (51/152) en middels SB's 16\% (24/152) (tab. 5). Dit verschil in detectie van $18 \%$ was significant $(p<0,001$; $95 \%-\mathrm{BI}=11-25 \%$ ). Een combinatie van SB's en TB's detecteerde significante prostaatkanker in $35 \%$ (53/152) van de gevallen, wat resulteerde in een verschil in prostaatkankerdetectie van $1 \%$ ten opzichte van TB's alleen. De sensitiviteit voor de detectie van prostaatkanker van TB's 
Tabel 4 Vergelijking uitkomsten van drie TB-technieken per PIRADS-score

\begin{tabular}{|c|c|c|c|c|}
\hline & FUS-TB & COG-TB & MRI-TB & \\
\hline \multicolumn{5}{|c|}{ detectiepercentage prostaatkanker, No. (\%) } \\
\hline PIRADS $3(n=64)$ & $6(26,1)$ & $5(23,8)$ & $5(25,0)$ & $p=0,99$ \\
\hline PIRADS $4(n=101)$ & $12(35,3)$ & $7(21,9)$ & $17(48,6)$ & $p=0,07$ \\
\hline PIRADS $5(n=69)$ & $21(95,5)$ & $22(88)$ & $20(90,9)$ & $p=0,66$ \\
\hline \multicolumn{5}{|c|}{ detectiepercentage sign. prostaatkanker ${ }^{\text {a }}$ No. (\%) } \\
\hline PIRADS $3(n=64)$ & $2(8,7)$ & $5(23,8)$ & $4(20,0)$ & $p=0,38$ \\
\hline PIRADS $4(n=101)$ & $7(20,6)$ & $5(15,6)$ & $9(25,7)$ & $p=0,60$ \\
\hline PIRADS $5(n=69)$ & $18(81,8)$ & $16(64,0)$ & $12(54,5)$ & $p=0,15$ \\
\hline
\end{tabular}

Sign significante

${ }^{\text {a }}$ Gleason-score $\geq 3+4=7$

Tabel 5 Vergelijking uitkomsten van TB's en SB's

\begin{tabular}{|c|c|c|c|c|c|}
\hline & & \multicolumn{3}{|c|}{ systematische biopten (SB's) } & \multirow[t]{2}{*}{ totaal } \\
\hline & & $\begin{array}{l}\text { geen prostaatkan- } \\
\text { ker }\end{array}$ & $\begin{array}{l}\text { insignificante pros- } \\
\text { taatkanker } \\
(\text { Gleason-score } \\
3+3=6)\end{array}$ & $\begin{array}{l}\text { significante prostaat- } \\
\text { kanker } \\
\text { (Gleason-score } \\
\geq 3+4=7 \text { ) }\end{array}$ & \\
\hline \multirow{3}{*}{$\begin{array}{l}\text { targetbiopten } \\
\text { (TB's) }\end{array}$} & geen prostaatkanker & $71(46,7)$ & $8(5,3)$ & $2(1,3)$ & 81 \\
\hline & $\begin{array}{l}\text { insignificante prostaat- } \\
\text { kanker (Gleason-score } \\
3+3=6 \text { ) }\end{array}$ & $12(7,9)$ & $8(5,3)$ & $0(0,0)$ & 20 \\
\hline & $\begin{array}{l}\text { significante pros- } \\
\text { taatkanker (Gleason- } \\
\text { score } \geq 3+4=7 \text { ) }\end{array}$ & $20(13,2)$ & $9(5,9)$ & $22(14,5)$ & 51 \\
\hline totaal & & 103 & 25 & 24 & $n=152$ \\
\hline
\end{tabular}

is 0,96 en van SB's is deze 0,45 ; de specificiteit voor de detectie van prostaatkanker is 0,78 en 0,98 respectievelijk.

Als SB's geen prostaatkanker detecteerden, werd door middel van TB's bij $21 \%(n=32)$ van deze patiënten wel prostaatkanker gedetecteerd, die in $13 \%(n=20)$ van de gevallen klinisch significant was. Als TB's geen prostaatkanker detecteerden, werd met SB's prostaatkanker gedetecteerd bij $7 \%(n=10)$ van de patiënten en significante prostaatkanker bij $1 \%(n=2)$ van de patiënten. In de totale groep $(n=152)$ werd klinisch significante prostaatkanker door TB's gemist in 1\% van de gevallen en door SB's in $19 \%$ van de gevallen. Dit verschil van $18 \%$ was significant $(p<0,001 ; 95 \%-\mathrm{BI}=11-24)$.

\section{Discussie}

Dit is de eerste gerandomiseerde multicenter studie in de internationale literatuur die de uitkomsten van drie TB-technieken met elkaar vergelijkt bij patiënten na negatieve SB's en een blijvende verdenking op prostaatkanker. Op basis van de resultaten van deze studie zijn er tussen de drie TBtechnieken geen significante verschillen in de detectiepercentages van prostaatkanker en significante prostaatkanker. Wel is het aantal benodigde biopten om deze gelijkwaardige detectie te bereiken, in de MRI-TB-groep het laagst (me- diaan 2 t.o.v. mediaan 4 in de FUS-TB- en 3 in de COGTB-groep). Het is natuurlijk de vraag hoe klinisch relevant deze laatste bevinding is, aangezien het risico op complicaties verwaarloosbaar is bij een mediane stijging van twee biopten.

De subanalyse van SB's en TB toont een significante toename van detectie van prostaatkanker en significante prostaatkanker in het voordeel van TB. Het toevoegen van SB's aan TB leidt niet tot een klinisch relevante stijging in detectiepercentages van prostaatkanker en significante prostaatkanker. Met andere woorden, het achterwege laten van herhaalde SB's in dit cohort leidde slechts tot het missen van $1 \%(n=2)$ klinisch significante prostaatkanker.

De 431 patiënten met een negatieve MRI (PIRADS $\leq 2)$ ondergingen biochemische follow-up. Ten tijde van deze analyse werd bij slechts $9(2 \%)$ patiënten prostaatkanker vastgesteld van wie $2(0,5 \%)$ na een mediane follow-up van 12 maanden significante prostaatkanker hadden. Gezien de beperkte follow-up van dit cohort moeten deze resultaten met enige terughoudendheid worden geïnterpreteerd. Een complete analyse van dit mpMRI-negatieve cohort volgt na langere follow-up.

Een meta-analyse van onze eigen onderzoeksgroep die werd gepubliceerd in 2017 en die eveneens de drie technieken van TB vergeleek, toonde een significant voordeel voor MRI-TB ten opzichte van COG-TB bij prostaatkan- 
kerdetectie [11]. Er waren geen significante verschillen tussen MRI-TB en FUS-TB, noch tussen FUS-TB en COGTB. Met betrekking tot significante prostaatkankerdetectie werden geen statistisch significante verschillen gevonden tussen de drie technieken. Het aantal studies dat meerdere technieken binnen één cohort rechtstreeks met elkaar vergeleek was zeer beperkt ten tijde van de meta-analyse. De recentere studies die meerdere technieken binnen één cohort rechtstreeks met elkaar vergeleken, toonden geen significante verschillen aan tussen de drie technieken, hetgeen in overeenstemming is met onze eigen studieresultaten [12-14].

De significante verbetering in de detectie van zowel prostaatkanker als significante prostaatkanker middels TB, in een cohort patiënten met eerdere negatieve SB's, is in overeenstemming met de uitkomsten van de review van Schoots et al. [15]. Filson et al. toonden in een gemixte populatie (bioptnaïef, eerdere negatieve SB's en AS) dat een combinatie van SB's en TB meer (significante) prostaatkanker vindt ten opzichte van elke methode afzonderlijk [16]. In onze studie is uitsluitend gekeken naar patiënten met eerdere negatieve SB's en in dit cohort is de toegevoegde waarde van het herhalen van SB's naast TB zeer beperkt.

De belangrijkste beperking van deze studie betreft de power ervan. Op basis van de literatuur ten tijde van het opstellen van het protocol werd ingeschat dat $69 \%$ van de patiënten een relevante afwijking zou vertonen op mpMRI [9]. Binnen ons eigen cohort is dit echter een aanzienlijk lager percentage $(35 \%)$. Hierdoor was slechts de helft van het initieel ingeschatte antal patiënten beschikbaar voor TB. De studie heeft daardoor een te lage power voor de primaire uitkomstmaat, hetgeen maar beperkt gecompenseerd wordt door de hoger dan verwachte detectiepercentages van de diverse technieken. De verschillen in detectiepercentages van significante prostaatkanker zijn echter dermate klein $(0,8-1,7 \%)$ dat zelfs bij een aanzienlijk groter cohort deze verschillen waarschijnlijk niet statistisch significant zouden zijn.

In onze studie is de primaire uitkomstmaat prostaatkankerdetectie. Voor de praktijk zou detectie van klinisch significante prostaatkanker een relevantere uitkomstmaat zijn geweest. Helaas is er momenteel geen eenduidige definitie van klinisch significante prostaatkanker in de setting van targetbiopten. In de meest recente literatuur wordt elke Gleason-score $\geq 3+4=7$ gebruikt als definitie van significante prostaatkanker, een definitie die ook wij hebben gehanteerd als secundaire uitkomstmaat [16]. Onze conclusies blijven overigens overeind bij het hanteren van een alternatieve definitie van klinisch significante prostaatkanker (gebaseerd op Gleason-score, PSA-density, tumorvolume en klinisch stadium) [1].

Een bekend fenomeen bij prostaatkankerdiagnostiek is de interobserver/operatorvariabiliteit die kan optreden bij de beoordeling van mpMRI's, het nemen van TB en de histopathologische analyse van biopten. Het feit dat onze onderzoeksgroep bestaat uit een team van dedicated radiologen, urologen en pathologen met betrekking tot prostaatkankerdiagnostiek, maakt dat de generaliseerbaarheid van de uitkomsten van gerichte biopten naar de algemene $\mathrm{Ne}$ derlandse praktijk mogelijk beperkt is. Er is onmiskenbaar een leercurve bij het verrichten van TB, die door alle biopteurs in iedere groep was doorlopen alvorens zij in het kader van de studie biopten gingen nemen.

Tot slot kon niet worden geblindeerd voor suspecte laesies bij het verrichten van SB's, gezien dit primair een studie was die drie technieken van TB vergeleek. Hierdoor was het noodzakelijk om bij de procedures te starten met TB om deformatie als gevolg van SB's te voorkomen. De effecten van het niet blinderen hebben we zo veel mogelijk proberen te beperken door een gestandaardiseerd schema voor SB's te gebruiken.

\section{Conclusie}

Bij patiënten met negatieve SB's en een blijvende klinische verdenking op prostaatkanker wordt in $35 \%$ van de gevallen een relevante afwijking op mpMRI gezien. In onze studie wordt geen significant voordeel gevonden voor één specifieke TB-techniek voor het detecteren van (significante) prostaatkanker in een populatie patiënten met eerder negatieve SB's. TB detecteerden significant meer (significante) prostaatkanker dan SB's binnen dit cohort. Gezien de beperkte toegevoegde waarde van SB's aan TB in deze populatie, kunnen SB's waarschijnlijk veilig achterwege gelaten worden.

Open Access This article is distributed under the terms of the Creative Commons Attribution 4.0 International License (http:// creativecommons.org/licenses/by/4.0/), which permits unrestricted use, distribution, and reproduction in any medium, provided you give appropriate credit to the original author(s) and the source, provide a link to the Creative Commons license, and indicate if changes were made. 


\section{Appendix 1}

Tabel 6 PIRADS v2

\begin{tabular}{ll}
\hline PIRADS 1 & Zeer laag: aanwezigheid klinisch significante prostaatkanker is zeer onwaarschijnlijk \\
PIRADS 2 & Laag: aanwezigheid klinisch significante kanker is onwaarschijnlijk \\
PIRADS 3 & Intermediair: aanwezigheid van klinisch significante kanker is onduidelijk \\
PIRADS 4 & Hoog: klinisch significante kanker is waarschijnlijk aanwezig \\
PIRADS 5 & Zeer hoog: klinisch significante kanker is zeer waarschijnlijk aanwezig \\
\hline
\end{tabular}

\section{Appendix 2}

a

sv<smiles>C1CCCCCC2(CCCC1)CCCCCCC2</smiles>

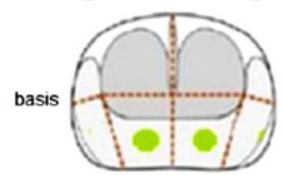

mid

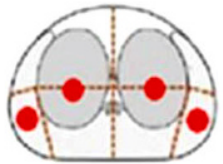

apex

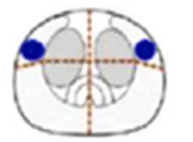

L

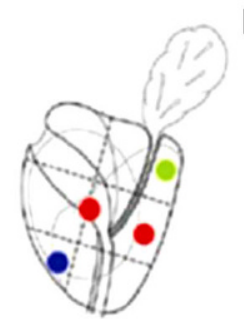

b

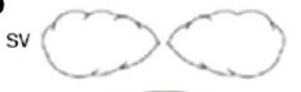

basis \begin{tabular}{c|c|c|}
\hline \\
\hline 1
\end{tabular}

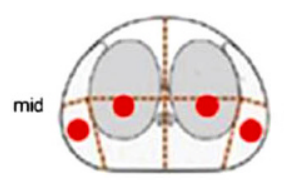

apex

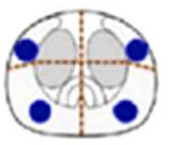

L

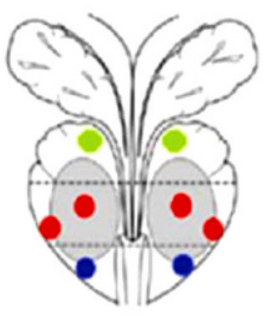

C
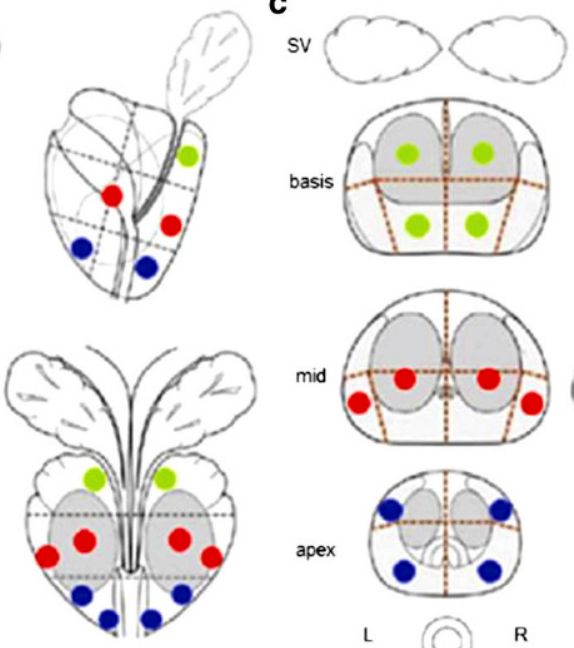

L
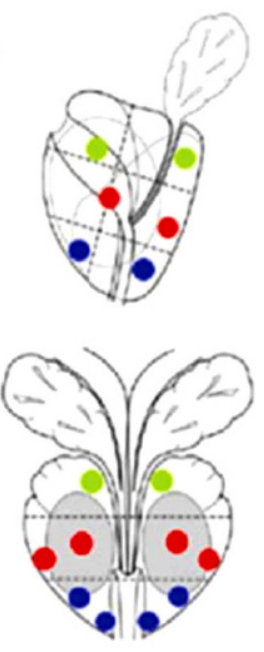

Tabel 6 Schema systematische biopten [5] a Prostaatvolume $<40 \mathrm{ml}$. b Prostaatvolume 40-60 ml. c Prostaatvolume $>60 \mathrm{ml}$ 


\section{Literatuur}

1. www.cijfersoverkanker.nl/prostaatkanker. Geraadpleegd op 28 november 2018.

2. www.oncoline.nl/prostaatcarcinoom. Geraadpleegd op 28 november 2018.

3. Heijmink SW, Moerkerk $H$ van, Kiemeney LA, Witjes JA, Frauscher F, Barentsz JO. A comparison of the diagnostic performance of systematic versus ultrasound-guided biopsies of prostate cancer. Eur Radiol. 2006;16:927-38.

4. Weinreb JC, Barentsz JO, Choyke PL, et al. PI-RADS Prostate Imaging - Reporting and Data System: 2015, Version 2. Eur Urol. 2016;69:16-40.

5. Barentsz JO, Richenberg J, Clements R, et al. ESUR prostate MR guidelines 2012. Eur Radiol. 2012;22:746-57.

6. Epstein JI, Allsbrook WC Jr, Amin MB, et al. The 2005 International Society of Urological Pathology (ISUP) Consensus conference on Gleason grading of prostatic carcinoma. Am J Surg Pathol. 2005;29:1228-42.

7. Welch HG, Fisher ES, Gottlieb DJ, Barry MJ. Detection of prostate cancer via biopsy in the Medicare-SEER population during the PSA era. J Natl Cancer Inst. 2007;99:1395-400.

8. Overduin CG, Futterer JJ, Barentsz JO. MRI-guided biopsy for prostate cancer detection: a systematic review of current clinical results. Curr Urol Rep. 2013;14:209-13.

9. Moore CM, Robertson NL, Arsanious N, et al. Image-guided prostate biopsy using magnetic resonance imaging-derived targets: a systematic review. Eur Urol. 2013;63:125-40.

10. Wegelin O, Melick HH van, Hooft L, et al. Comparing three different techniques for magnetic resonance imaging-targeted prostate biopsies: a systematic review of in-bore versus magnetic resonance imaging-transrectal ultrasound fusion versus cognitive registration. Is there a preferred technique? Eur Urol. 2017;71:517-31.

11. Yaxley AJ, Yaxley JW, Thangasamy IA, et al. Comparison between target magnetic resonance imaging (MRI) in-gantry and cognitively directed transperineal or transrectal-guided prostate biopsies for Prostate Imaging-Reporting and Data System (PI-RADS) 3-5 MRI lesions. BJU Int. 2017;120:43-50.

12. Arsov C, Rabenalt R, Blondin D, et al. Prospective randomized trial comparing magnetic resonance imaging (MRI)-guided in-bore biopsy to MRI-ultrasound fusion and transrectal ultrasound-guided prostate biopsy in patients with prior negative biopsies. Eur Urol. 2015;68:713-20.

13. Kaufmann S, Russo GI, Bamberg F, et al. Prostate cancer detection in patients with prior negative biopsy undergoing cognitive-, robotic- or in-bore MRI target biopsy. World J Urol. 2018;36:761-8.

14. Schoots IG, Roobol MJ, Nieboer D, et al. Magnetic resonance imaging-targeted biopsy may enhance the diagnostic accuracy of significant prostate cancer detection compared to standard transrectal ultrasound-guided biopsy: a systematic review and meta-analysis. Eur Urol. 2015;68:438-50.

15. Filson CP, Natarajan S, Margolis DJ, et al. Prostate cancer detection with magnetic resonance-ultrasound fusion biopsy: the role of systematic and targeted biopsies. Cancer. 2016;122:884-92.

16. Kasivisvanathan V, Rannikko AS, Borghi M, et al. MRI-targeted or standard biopsy for prostate-cancer diagnosis. N Engl J Med. 2018;378:1767-77.

drs. O. Wegelin arts-assistent urologie

drs. L. Exterkate arts-onderzoeker urologie

prof. dr. J.O. Barentsz radioloog

drs. M.G. van der Leest radioloog

drs. J.C. Kelder epidemioloog

prof. dr. J.A. Kummer patholoog

dr. W. Vreuls patholoog

dr. P.C. de Bruin patholoog

drs. T.F. de Vocht uroloog

dr. J.E. Nuininga uroloog

prof. dr. J.L.H.R. Bosch uroloog

dr. D.M. Somford uroloog

dr. H.H.E. van Melick uroloog 